\title{
Fault-Tolerant Actuation Architectures for Unmanned Aerial Vehicles
}

\author{
M. A. A. Ismail, C. Bosch, S. Wiedemann, and A. Bierig
}

\begin{abstract}
Rising civilian applications that make use of unmanned aerial vehicles (UAVs) demand crucial precautions to minimize safety hazards. Future UAVs are expected to incorporate fault-tolerant architectures for critical on-board systems to ensure compliance with airworthiness certification. Reliability reports of in-service UAVs showed that flight control actuators are among the highest root-causes of UAVs mishaps. In this paper, the current state-of-the-art actuation architectures for UAVs are reviewed to identify technical requirements for certification. This work is part of a TEMA-UAV research project aimed at developing certifiable fault-Tolerant Electro-Mechanical Actuators for future UAVs.
\end{abstract}

Keywords Airworthiness certification for UAVs - Electro-mechanical actuators • Fault-tolerant control

\section{Introduction}

Unmanned aerial vehicles (UAVs) have provided superior performance for many civil and military applications due to their low acquisition and operating costs. However, the reliability level of in-service UAVs is not equivalent to general aviation, according to several reliability reports based on about 290,000 flight hours (FH) [1-3]. The US Office of the Secretary of Defense has stated, "improving UA reliability is the single most immediate and long-reaching need to ensure their success" [1]. UAVs currently have a loss rate 10 times worse than manned aircraft category, signaling that the

M. A. A. Ismail $(\varangle) \cdot$ A. Bierig

DLR (German Aerospace Center), Institute of Flight Systems, Lilienthalplatz 7, 38108

Brunswick, Germany

e-mail: Mohamed.Ismail@dlr.de

C. Bosch

Institute of Helicopter Technology, Technical University of Munich, Arcisstraße 21, 80333

Munich, Germany

S. Wiedemann

MACCON Elektroniksysteme GmbH, Aschauer Str. 21, 81549 Munich, Germany

(C) Springer Nature Singapore Pte Ltd. 2021

L. Gelman et al. (eds.), Advances in Condition Monitoring and Structural

Health Monitoring, Lecture Notes in Mechanical Engineering,

https://doi.org/10.1007/978-981-15-9199-0_32 


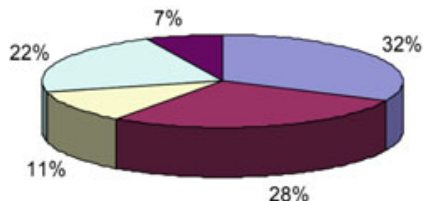

IAI fleet

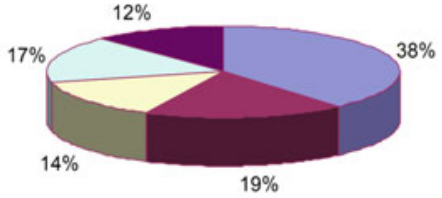

US army fleet

Fig. 1 Average source of system failures for in-service UAVs fleets [1]. IAI = Israeli Aircraft Industries

root-causes of these losses should be identified and mitigated [3]. Here, the scope is for UAVs of a takeoff-weight $>25 \mathrm{~kg}$ because of their certification potential [4]. System failures for two large fleets (the US army and Israeli Aircraft Industries) are shown in Fig. 1, in which flight control systems are the second highest root-cause of UAVs failures.

Flight control system failures (Fig. 1) have been investigated to the following findings $[1,2]$ :

- Flight control systems failures are usually failures of the electro-mechanical actuators (EMAs).

- The root-causes of EMA failures within US army fleets can be grouped into:

- EMAs designed for neither aerospace nor UAVs (e.g., Pioneer UAVs, with a loss rate of 334 per 100,000 FH compared to 1 per 100,000 FH for general aviation); and

- EMAs that partially involve components matching the manned aircraft category (e.g., Predator UAVs with a loss rate of 32 per 100,000 FH).

The superior reliability level of the manned aircraft category is maintained by certification requirements that are being extended to UAVs [4]. Recently, the European Aviation Safety Agency published safety regulations for UAV s considering three UAV categories: open (takeoff-weight $<25 \mathrm{~kg}$ ), specific, and certified. A detailed discussion of the certification requirements for different UAVs is provided in a followup paper [4]. In this paper, the scope is for flight control actuators that are directly influenced by the expected certification conditions for UAVs:

C1. No single failure will result in a catastrophic-failure condition (evaluated by an UAV level functional hazard analysis); and

C2. Each catastrophic-failure condition is extremely improbable.

The first condition implies a fault-tolerant control (FTC) design, as the flight control function must not be interrupted after the first failure [5]. The second condition relates to a quantitative probability of occurrence for possible catastrophic scenarios. Two FTC approaches have been cited [6-9] for mitigating flight control actuator failures: the aircraft and actuator FTCs shown in Fig. 2.

The aircraft FTC is implemented in the flight control computer (FCC), which activates modified flight control laws to compensate for actuator failures, as described in 


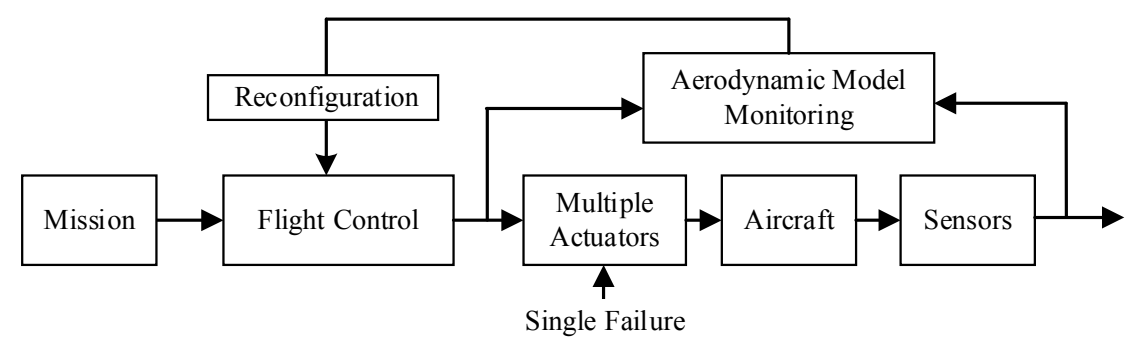

Aircraft based FTC for actuator failures

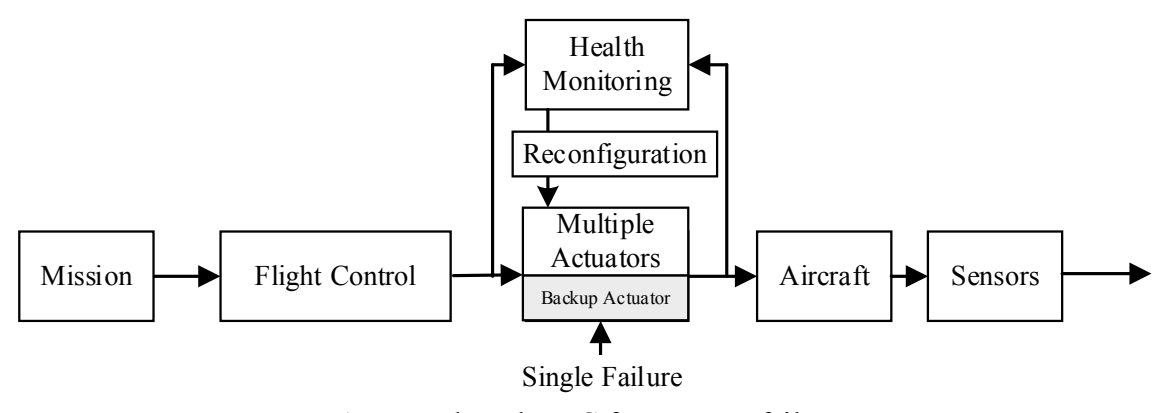

Actuator based FTC for actuator failures

Fig. 2 FTCs for accommodating UAVs actuator failures

[6, 7]. In [6], a multi-variable FTC was developed for controlling a fixed-wing UAV with a defective actuator, using only one operational control surface. In [7], a FTC with a bank of observers was used to compensate for jammed flight control actuators, but was limited to small jammed angles, making it insufficient for certification requirements. The FTC actuation architectures are based on subsystem redundancies and health monitoring functions $[8,9]$. In this paper, the current state-of-the-art FTC actuation technologies and architectures for UAVs are reviewed in Sects. 2 and 3 , respectively. The technical challenges for airworthiness certification and a new electrical fault-tolerant topology are discussed in Sect. 4.

\section{Fault-Tolerant Technologies}

\subsection{Electrical Motors}

Several electrical motor technologies have been evaluated briefly in the literature for flight control actuators [9-14]. Among those technologies are machines without magnets, such as induction machines (IMs), switched reluctance machines (SRMs) and synchronous reluctance machines (SynRMs), and machines with permanent magnets (PMs), such as surface-mounted permanent-magnet synchronous machines 
(SPMSM or PMSM) and interior permanent-magnet synchronous machines (IPMSM). IMs are known for their ruggedness, reliability, and inexpensive cost, but they provide limited power-to-weight density [9]. SRMs are considered more widely in fault-tolerant applications due to their multiple single-phase winding configurations and their inherent capability to continue the operation after a phase fault. However, they also exhibit comparatively high torque ripples (especially during a phase loss), have comparatively higher acoustic noise, and a reduced power density of approximately 30-50\% compared to SPMSMs [10, 11]. SynRMs use distributed three-phase windings, allowing synchronous rotor operation with the stator magnetic field. Their torque ripples are comparative to the SPMSM and IPMSM while their power density lies between the SPMSM and the IM [12] and is strongly dependent on the rotor geometry.

PM-free machines have the advantages of no cogging- or drag torque (in case of a short circuit failure), lower cost, easier manufacture, and greater robustness against mechanical and temperature shocks. Due to their low back-electromotive force, these machines lead to better inverter control failsafe operation, since the inverter does not need to deal with uncontrollable rectification, even at high speed [13]. PM machines, by contrast, are widely used as flight control actuators because of their superior power-to-weight density and their relatively simple control techniques $[9,14]$. The IPMSM has, in comparison with the SPMSM, a higher reluctance torque and, therefore, a higher power density. Nevertheless, PM machines have, besides cogging effects (if the rotor or stator is not skewed), several fault-tolerant related drawbacks, such as drag torque (caused by short circuit faults), electrical and thermal phase coupling (triggered by distributed windings), magnet (hysteresis) losses, and comparatively high back-electromotive force. PMs are also prone to change over time as a function of temperature stresses (aging) and field weakening operation, as well as speed limitation if the magnets are surface-mounted instead of buried (e.g., SPMSM). The permanent-magnet-assisted synchronous reluctance machine (PMa-SynRM) is a hybrid reluctance machine possessing PM elements partially inserted into the rotor flux barriers for increased power density and, consequently, increased manufacturing complexity [12]. Instead of rare-earth magnets, cheaper ferrites can be used, which leads to decreased power density and actuator temperature range. That being said, PMa-SynRMs enjoy the aforementioned PM-less advantages to a certain degree as well as higher torque-to-weight, in comparison with the SynRM, and an extended field weakening range compared to the SPMSM and IPMSM [13]. Efficiency and power density depend strongly on rotor geometry and inserted magnetic material. Therefore, it is scalable between the IPMSM and SynRM $[15,16]$. Due to design similarity, the reliability of the PMa-SynRM can be considered equivalent or better than the IPMSM, as less magnet material is used. Less magnet material also grants a lower drag torque compared to conventional PM machines in case of a short circuit fault. Consequently, and in a fault-tolerant context, conventional PM machines should be overrated from two to four times the nominal ratings [11] depending on low- or high-speed operation. Yet, because of lower drag torque and magnet material, the PMa-SynRM requires a smaller overrating, making it an interesting alternative to conventional PM and reluctance machines by size and weight. 


\subsection{Electrical Power Drive}

The power drive is responsible for controlling electric power and delivering it to the motor through power transistors (e.g., MOSFETs or IGBTs [14]). The arrangement of power transistors depends on the motor configuration, namely multiple independent three-phase modules (MITPMs) and multiple independent single-phase modules (MISPMs). MITPMs encompass one or more motors divided into sets of three-phase star-connected modules, each powered by three-phase transistor bridges. Rated power is supplied by $n$ sets of three-phase modules connected in parallel. For example, in the $3+3$ system shown in Fig. 3 (left), two sets of three-phase modules are redundant in one rotor $[9,17]$.

MISPMs encompass a single motor divided into electrically isolated $n$ phases, each powered by an H-bridge. Rated power is supplied by $n$ sets of H-bridge modules. For example, in the $2+1$ system shown in Fig. 3 (right), three sets of isolated single-phase modules are redundant in one rotor. The motor could be operated by 2 active phases (i.e., a faulty phase) using a modified pulse width modulation (PWM) waveforms [14]. The isolated phases may comprise three to six phases [8,9].

\section{Fault-Tolerant Actuation Architectures}

Fault-tolerant actuation architectures, A1-A6 in Fig. 4, comprise control units (CONs), monitoring units (MONs), position sensors for the actuator deflection angle, communication channels (COMs) to FCCs, clutches, and electric motors based on technologies in Sect. 2.1. All of these architectures include fault-tolerant electrical motors configured as dual motors (e.g., A1, A3, A4, and A5) or as single motors with backup windings (e.g., A2 and A6). The mechanical power of the dual electric motors is transferred to the control surface by using either a torque-summing gearbox (e.g., A3 and A4), an electric clutch (e.g., A5) or a directly controlled surface linkage (e.g.,
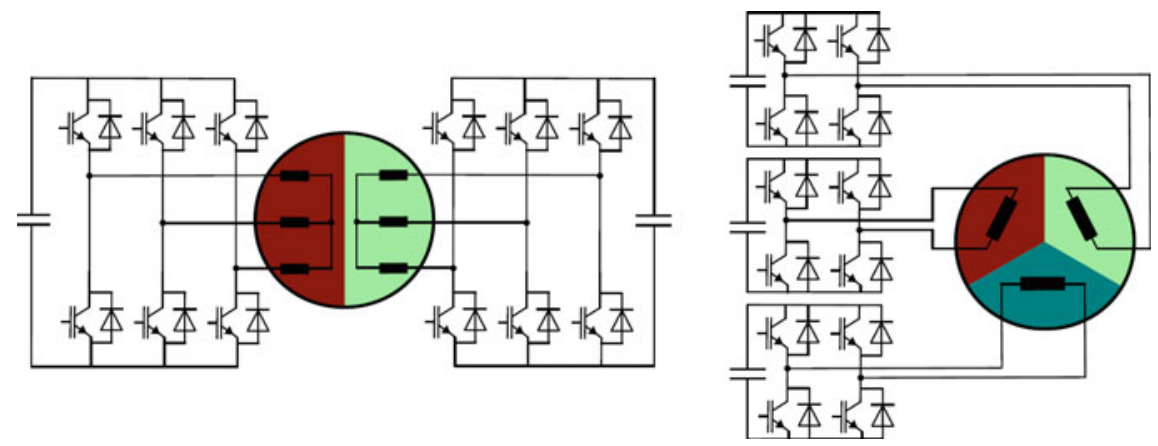

Fig. 3 Widely cited drive topologies for $3+3$ (left) and for $2+1$ (right) [9] 


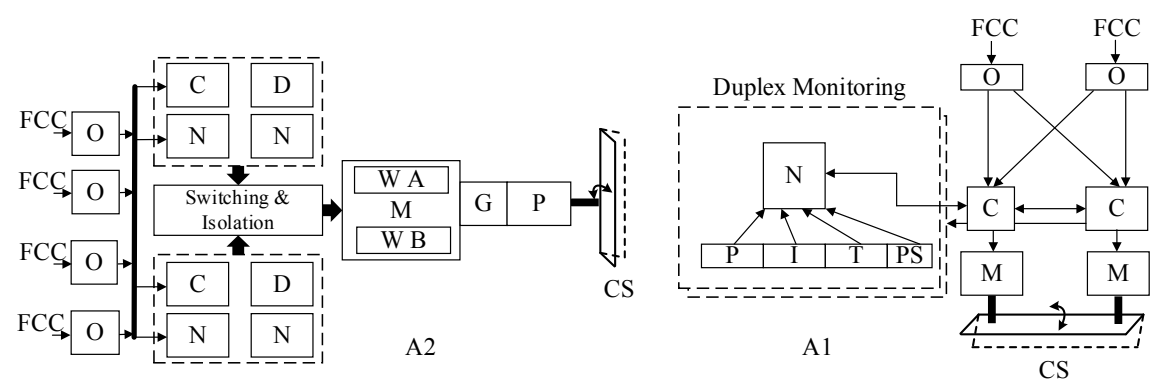

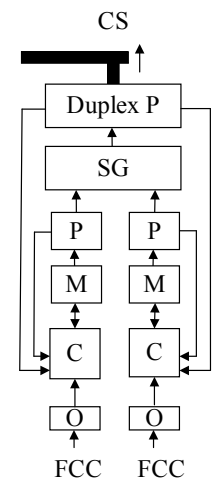

A3

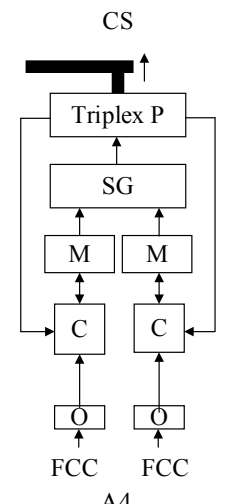

A4
CS

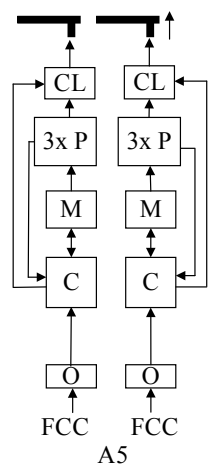

CS

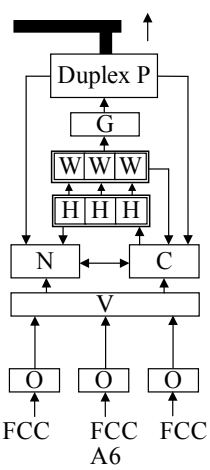

P Position sensor

M Motor

C Control unit

$\mathrm{V}$ Voter unit

N Monitoring unit

SG Torque summing gearbox CL Clutch

$\mathrm{O}$ Communication

Fig. 4 Examples of fault-tolerant actuation architectures for UAVs: A1 [20], A2 [21], A3 [22], A4 [23], A5 [18], A6 [8]

A1). Sensor redundancies are present in position sensors because of their high failure rates. A4 and A5 feature a typical duplex layout used for general purpose EMAs, such as those in many in-service UAVs, but they are not fault-tolerant in-flight. A6 involves a voter to reliably tolerate incorrect commands from triple FCCs, despite having only one control unit, making it insufficient to keep the actuator operational after its first failure. A5 was developed for optionally piloted vehicles (OPV, a category between UAVs and certified manned aircraft [18]). Further details on how A5 manages in-flight reconfiguration for optionally piloted vehicles are not available. 


\section{Discussion}

\subsection{Electrical Topology}

An evaluation of electrical topologies, including PMSMs and PMa-SynRMs, is shown in Fig. 5. The complexity is the number of power transistor (PT) switches (e.g., MOSFETs, multiplied by 4 for a better illustration). The overratings for individual power transistors in a SPMSM are calculated in [14], mainly comprising additional torque contributions for a partial loss of actuator torque by an open circuit lane (a single phase for MSPMs or a three-phase set for MTPMs) and a drag torque by a short circuit lane at low speeds. The overratings for PMa-SynRMs are estimated by assuming a reduction in drag torque only by a factor of 0.9 , according to the investigation in [19], while the overrating due to the open circuit faulty remains unchanged. Considering minimum complexity as a vital selection criterion for UAVs, topologies $2+1$ and $3+3$ are the best candidates within MISPMs and MITPMs, respectively. In a $3+3$ topology, the motor control provides ripple-free torque after losing an active lane. In a $2+1$ topology, the motor control must use asymmetric switching waveforms to keep the motor running with only two phases [9, 14]. These waveforms cause undesirably high levels of current harmonics, violating the total harmonic distortion requirements set by DO-160 [9] that may be considered for certification requirements. Therefore, a $3+3$ topology of PMa-SynRMs has good potential for certified fault-tolerant actuation for UAVs.

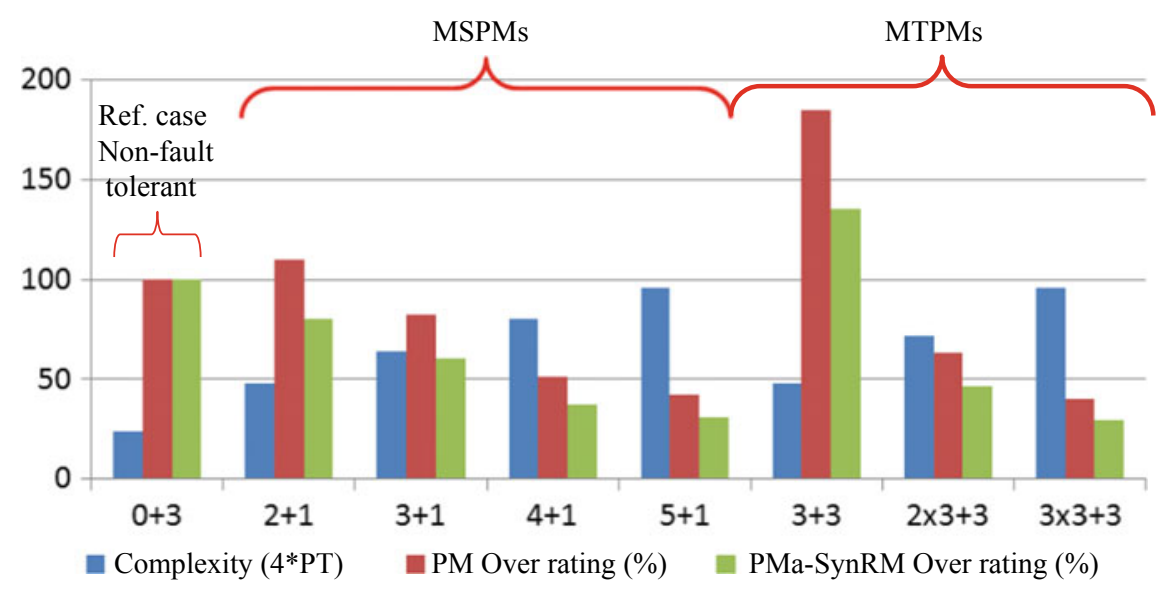

Fig. 5 Evaluations for different electrical topologies considering their complexities and over ratings 


\subsection{Architecture Characterization for Airworthiness Certification}

The UAV actuation architectures cited in this study can be characterized into three groups:

(1) Non-fault-tolerant actuation architectures

- Single-channel industrial actuators

- Actuator failures may be tolerated in FCCs by modified control laws.

Examples: Pioneer UAVs [2] and [6, 7]

(2) Partial fault-tolerant actuation architectures

- FCC communication channels are less than three, insufficient for tolerating a drift (a challenging fault) or incorrect FCC commands in general [8, 14].

- On-ground or a partial in-flight reconfiguration after a single critical failure.

Examples: A1 [11], A3 [13], A4 [14], and A5 [15].

(3) Full fault-tolerant actuation architectures

- Triplex communications applied for reliable FCC commands by a voter.

- All catastrophic-failure conditions to which the actuator and its controller are the main contributor or only source are mitigated by fail operational or failsafe modes.

- In-flight actuator reconfiguration after a single critical failure.

Example: A2 [12], however, neither a prototype nor test setup has been reported.

This group complies with $\mathrm{C} 1$ (Sect. 1), but C2 requires a reliability analysis discussed in a follow-up study [4].

\section{Conclusion}

The limited reliability of in-service UAVs has been significantly attributed to failures caused by flight control EMAs, because they are neither fully fault-tolerant nor qualified for UAVs. Recently, fault-tolerant actuators have been exploited by some commercial UAVs, comprising mainly fault-tolerant capabilities for electric motors and position sensors. These actuators are mostly based on PM motors to achieve a high power-to-weight density. However, PM motors should be overrated two to four times to compensate for expected electrical failures. Alternatively, the PMa-SynRM of $3+$ 3 drive topology has been discussed here, using less drag torque and magnet material to obtain a smaller overrating, making it an interesting alternative to conventional PM-based EMAs by size and weight. The term "fault-tolerant actuator" has been used frequently in the literature to describe EMAs that can partially maintain functionality 
after some critical failures. A fault-tolerant actuator for a certified UAV demands more rigorous requirements, namely: (a) all significant failures should be identified within a specific probability of occurrence as defined by a certification body; (b) these failures should be accommodated by fault detection and reconfiguration techniques; and (c) these techniques should be operational online during flight phases.

Acknowledgements This work was supported by the TEMA-UAV project through the German

National Aerospace Research Program (Lufo V-3).

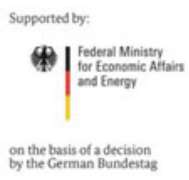

\section{References}

1. US Office of the Secretary of Defense (2005) Unmanned Aircraft Systems Roadmap 20052030. US Office of the Secretary of Defense, United States. https://fas.org/irp/program/collect/ uav_roadmap2005.pdf. Accessed 2005

2. Israel K, Nesbit R (2004) Defense science board study on unmanned aerial vehicles and uninhabited combat aerial vehicles. US Defense Science Board, United States

3. Sadeghzadeh I, Zhang Y (2011) A review on fault-tolerant control for unmanned aerial vehicles (UAVs). Infotech@Aerospace. p 1472

4. Bosch C et al (2019, under publication) Towards certifiable fault-tolerant actuation architectures for UAVs. In: WCCM 2019, Singapore

5. Lauffs JP, Holzapfel F (2016) Hardware-in-the-loop platform for development of redundant smart actuators. Aircraft Eng Aerosp Technol: Int J 88(3):358-364

6. Venkataraman R, Seiler PJ (2016) Safe flight using one aerodynamic control surface. In: AIAA guidance, navigation, and control conference, $\mathrm{p} 0634$

7. Bateman F, Noura H, Ouladsine M (2011) Active fault diagnosis and major actuator failure accommodation: application to a UAV. In: Advances in flight control systems, InTech 2011, pp 137-158

8. Di Rito G, Galatolo R, Schettini F (2016) Self-monitoring electro-mechanical actuator for medium altitude long endurance unmanned aerial vehicle flight controls. Adv Mech Eng 8(5):1687814016644576

9. Cao W et al (2011) Overview of electric motor technologies used for more electric aircraft (MEA). IEEE Trans Ind Electron 59(9):3523-3531

10. Radun AV (1992) High-power density switched reluctance motor drive for aerospace applications. IEEE Trans Ind Appl 28(1):113-119

11. Pickert V (2019) Fault Tolerant. ECPE, Leinfelden-Echterdingen, Germany

12. Donaghy-Spargo CM (2016) Synchronous reluctance motor technology: opportunities, challenges and future direction. Eng Technol Ref, pp 1-15

13. Wang B et al (2017) A fault-tolerant machine drive based on permanent magnet-assisted synchronous reluctance machine. IEEE Trans Ind Appl 54(2):1349-1359

14. Bennett JW (2010) Fault tolerant electromechanical actuators for aircraft. Ph.D. thesis, Newcastle University

15. Ibrahim M, Rashad E, Sergeant P (2017) Performance comparison of conventional synchronous reluctance machines and PM-assisted types with combined star-delta winding. Energies 10(10): 1500 
16. Torres López Torres C (2018) Analysis and implementation of a methodology for optimal PMa-SynRM design taking into account performances and reliability, Ph.D. thesis, Universitat Politècnica de Catalunya

17. Zhu J, Niu X (2010) Investigation of short-circuit fault in a fault-tolerant brushless permanent magnet ac motor drive with redundancy. In: 5th IEEE conference on industrial electronics and applications, pp 1238-1242

18. Technical manuals Pegasus GmbH, 10 December 2018. www.pegasus.de

19. Ullah $S$ et al (2016) A permanent magnet assisted switched reluctance machine for more electric aircraft. In: XXII ICEM, pp 79-85

20. Flurry SJ (2010) KR Patent No. 100972516B1

21. Clarkstone JK, Morgan S, White N (2012) Australian Patent No. 2012235920B2

22. Hadeokju (2016) KR Patent No. 101658459

23. Technical manuals Volz GmbH, 10 December 2018. www.volz.de 